HITLER IN DER OPER

Deutsches Musikleben 1919 - 1945 


\author{
Michael Walter
}

\title{
HITLER
}

\section{IN DER OPER}

Deutsches Musikleben 1919 - 1945

\section{Verlag J. B. Metzler Stuttgart - Weimar}


Die Deutsche Bibliothek - CIP-Einheitsaufnahme

\section{Walter, Michael:}

Hitler in der Oper : deutsches Musikleben 1919 - 1945 / Michael Walter.

- Stuttgart ; Weimar : Metzler, 2000

ISBN 978-3-476-01788-8

ISBN 978-3-476-01788-8 ISBN 978-3-476-02759-7 (eBook)

DOI 10.1007/978-3-476-02759-7

Dieses Werk einschließlich aller seiner Teile ist urheberrechtlich geschützt. Jede Verwertung außerhalb der engen Grenzen des Urheberrechtsgesetzes ist ohne Zustimmung des Verlages unzulässig und strafbar. Das gilt insbesondere für Vervielfältigungen, Übersetzungen, Mikroverfilmungen und die Einspeicherung und Verarbeitung in elektronischen Systemen.

(C) 2000 Springer-Verlag GmbH Deutschland Ursprünglich erschienen bei J. B. Metzlersche Verlagsbuchhandlung und Carl Ernst Poeschel Verlag GmbH in Stuttgart 1995/2000 


\section{N H A L T}

Vorwort

Die Mörder sitzen im Rosenkavalier

Die Neuorientierung des deutschen Musiklebens

nach dem ersten Weltkrieg

Von Friedrich Ebert zum Kampfbund für deutsche Kultur

Bedingungen und Voraussetzungen der Gleichschaltung

bürgerlicher Musikkultur in einer Kleinstadt

Oper 1918-1933

Die ideelle Tatsächlichkeit der Oper

Meyerbeers Opern in der Weimarer Republik

Hitler in der Oper

Der »Führer« und Werner Egks Peer Gynt

Die Vermählung einer idealen Politik mit einer realen Kunst Oper und Musikpolitik im Dritten Reich

Volksgemeinschaft in der Oper

Ottmar Gersters Hexe von Passau

Die Musik des Olympiafilms von 1938 


\section{Vorwort}

Die "Kultur der Weimarer Republik" ist ein Schlagwort, mit dem keineswegs die Kultur der Weimarer Republik, sondern jener Teil, der uns heute für diese Republik typisch gewesen zu sein scheint, zusammengefaßt wird. Nur wenn man die Kultur der Weimarer Republik auf diesen Teilbereich verengt, scheint es, daß die "Nationalsozialisten alle Spuren, die an diese Ära erinnerten, aufs gründlichste $\mathrm{zu}$ tilgen versucht ${ }^{\mathrm{m1}}$ hatten. Man braucht (in einem gängigen Argumentationsmuster), im Hinblick auf die Musikkultur nach 1933, nur die neue Musik zu erwähnen, sie mit dem Terminus der »entarteten Musik« zu korrelieren und auf die emigrierten Komponisten hinzuweisen, um einen sinnfälligen Bruch zwischen dem Musikleben in der Weimarer Republik und dem nationalsozialistischen Regime zu demonstrieren. Ein solcher Bruch ist aber nichts anderes als eine historiographische Inszenierung, wie schon die Tatsache zeigt, daß der von den Nationalsozialisten verpönte Jazz zunächst weder umgehend generell verboten noch mit allen Mitteln bekämpft wurde ${ }^{2}$, vielmehr verwendete man nicht unerhebliche Anstrengungen auf die Etablierung einer Art 'reichsdeutscher' Jazz-Variante. Auch die neue Musik und die Zeitopern von Kurt Weill oder Ernst Krenek beeinflußten die Komponisten bis in die dreißiger und vierziger Jahre, was nicht verwunderlich ist, wenn man bedenkt, daß gerade die besseren Komponisten des Dritten Reichs weder ignorieren noch umstandslos negieren konnten, was sich musikgeschichtlich in den zwanziger Jahren ereignet hatte. Reduziert man jedoch die Musikgeschichte der zwanziger und dreißiger Jahre auf die neuen Aspekte der Kompositionsgeschichte, verkennt man, indem man rückwirkend pointiert, was kompositionsgeschichtlich nach 1945 wirkungsmächtig war, die Realität des Musiklebens jener Zeit. Für das Opernpublikum der letzten Jahre der Republik spielten nicht Schönberg, Berg oder Weill eine zentrale Rolle, sondern die chronologisch vergleichsweise 'neue' und als solche akzeptierte Musik wurde durch Giacomo Puccini und

1J. Hermand/F. Trommler, Die Kultur der Weimarer Republik, Frankfurt/M. 1988, 7.

${ }^{2}$ Vgl. H. Schroder, Tanz- und Unterhaltungsmusik in Deutschland 1918-1933, Bonn 1990 [= Orpheus-Schriftenreihe zu Grundfragen der Musik 58] u. M.H. Kater, Different Drummers. Jazz in the Culture of Nazi Germany, New York/Oxford 1992. - Um Mißverstăndnissen vorzubeugen, sei betont, daß es hier nicht um eine ideologische oder faktische 'Entlastung' des Nationalsozialismus geht, sondern nur um die Wahrnehmungsperspektive um die Mitte der dreißiger Jahre. 
Richard Strauss repräsentiert. An deren zentraler Bedeutung für das Opernleben änderte auch die Entwicklung nach 1933 nichts.

Die Frage, wie sich solche Kontinuitäten erklären lassen, steht ebenso hinter den hier vorgelegten Studien wie die Frage nach der Modernität, nicht nur der nationalsozialistischen, sondern auch der republikanischen Musikkultur. Wenn ich mich hierbei überwiegend der Oper zugewandt habe, ist dies darin begründet, daß sich die Oper als jene Gattung erweist, an der man am besten die Ambiguitäten, aber auch die Ziele des Musiklebens der Zeit der Weimarer Republik und des Nationalsozialismus demonstrieren kann. Das gilt sowohl hinsichtlich soziologischer wie ideologischer Strukturen.

Die einzelnen Aufsätze dieses Buchs beziehen sich nicht nur sachlich, sondern auch in der Fragestellung aufeinander. Ihnen allen liegt die These zugrunde, daß das Musikleben der Weimarer Republik sich wesentlich von dem der Kaiserzeit unterschied. Auch prägte es in vielem das Musikleben des Dritten Reichs und beeinflußte damit die Entscheidung von Musikern und MusikRezipienten für die 'Kultur' des Dritten Reichs bzw. den mangelnden Widerspruch gegen diese. Der historische Bruch ist also nicht erst 1933, sondern bereits in den Jahren 1918 bis 1920 feststellbar ${ }^{3}$. Dabei ist allerdings zu betonen, daß, was für die politische Entwicklung in der Weimarer Republik gilt, auch für die kulturelle Entwicklung Geltung hat, nämlich daß sie "nicht als bloße Vorgeschichte des Dritten Reiches interpretiert werden" kann $^{4}$ (eher schon ist die Musikkultur des Dritten Reichs der Nachklang der republikanischen).

Während das Musikleben an der nationalen Aufbruchstimmung nach dem ersten Weltkrieg partizipierte, stand es der »totalen Revolution«, die Goebbels

\footnotetext{
${ }^{3}$ Wenn dieser Bruch betont wird, bedeutet dies nicht, daß "longue duree"-Effekte ausgeschlossen wăren, wie sie z.B. B. Sponheuer (Musik, Faschismus, Ideologie. Heuristische Uberlegungen, in: Die Musikforschung 46 [1993], 241-253) seinen Überlegungen zugrunde legt. Aber dort, wo solche Effekte deutlich werden (vgl. z.B. die Studie über das Musikleben in einer Kleinstadt in diesem Band), wird zugleich deutlich, daß sie nur in der Brechung durch die neue gesellschaftliche und politische Entwicklung, d.h. in der Auseinandersetzung mit dieser wahrgenommen werden. Zudem ist nicht der Rückgriff auf das 19. Jahrhundert signifikant, sondern dessen Scheitern (wofür sowohl Furtwängler als auch Richard Strauss gute Beispiel abgeben). Es handelt sich hier weniger um ein Problem der Ideengeschichte als um ein generationstypisches Problem, das gerade dort auftrat, wo politische Probleme eher unterschwellig denn manifest vorhanden waren bzw. wahrgenommen wurden.
}

${ }^{4} \mathrm{H}$. Mommsen, Die verspielte Freiheit. Der Weg der Republik von Weimar in den Untergang 1918 bis 1933, Frankfurt/Berlin 1990, 9. 
immer im Munde führte, in merkwürdig indifferenter Weise gegenüber, die letztlich jedoch vielfach in Opportunismus mündete. Das galt auch im Hinblick auf den Antisemitismus der Nationalsozialisten; gerade dessen Auswirkungen wurden von einem Großteil der Musiker - Richard Strauss mag hier als Beispiel stehen - wenn nicht begrüßt, so doch durchaus geduldet und akzeptiert. Aber eine wirkliche Musikpolitik, im emphatischen Sinne des Worts - über die Durchführung rassistischer Maßnahmen und die Funktionalisierung vor allem der populären Musik als Propagandainstrument hinaus - hat es im Dritten Reich nicht gegeben. Es gelang nicht, das Gerede vom »Neubau deutscher musikalischer Kultur " (Peter Raabe) in die Tat umzusetzen, weder hinsichtlich des Musiklebens, also neuer Darbietungsformen der Musik, noch hinsichtlich der Kompositionen: im 'besten Falle' waren sie, wie Werner Egks Peer Gynt, kompositionsgeschichtlich der Republik verpflichtet, oder aber sie taugten wenig (wie Ottmar Gersters Hexe von Passau). Beides aber - der Rekurs auf Elemente der zwanziger Jahre ebenso wie die mangelnde kompositorische Qualität war Anzeichen dafür, daB von einer, von den Machthabern vielbeschworenen, spezifisch nationalsozialistischen »Modernität" nicht die Rede sein konnte; andererseits aber kann das Musikleben nach 1933 auch nicht mit dem Terminus der bloßen "Reaktion" - wie sie sich etwa in singspielähnlichen Kompositionen im Stile Lortzings hätte erweisen können - charakterisiert werden. Genaueres Hinsehen erweist, daß einer der Gründe für die mangelnde Konsistenz und die fehlenden Resultate der Musikpolitik die ideologischen Querelen zwischen Propagandaministerium und "Amt Rosenberg" waren, die sowohl im 'Fall Hindemith' wie im 'Fall Egk' durch Hitler selbst entschieden wurden. Gerade Hitler entsprach aber einem bestimmten Typus des Opernbesuchers in den zwanziger Jahren und steht insofern selbst für einen Kontinuitäts-Aspekt des Musiklebens nach 1933.

Natürlich änderten sich mit der Machtübernahme der Nationalsozialisten viele Rahmenbedingungen des Musiklebens, aber nach Ansicht vieler Zeitgenossen änderten sie sich zum Besseren hin. Nationalsozialistische Kulturpolitiker boten scheinbar einer weit über die Parteigänger hinausreichenden Öffentlichkeit und vielen Protagonisten an, was diese bereits in der Republik gefordert hatten. Das läßt sich gerade am Musikleben einer Kleinstadt sinnfällig machen, in dem der Übergang von der Republik in die Diktatur als positive Zäsur empfunden wurde. Aber auch Richard Strauss kann hier als Beispiel stehen, denn die Nationalsozialisten gaben ihm, was ihm die Republik 1919, als er Intendant der Berliner Staatsoper werden wollte, aus guten Gründen und hellsichtig verweigert hatte: ein Amt, in dem er das deutsche Musikleben und 
die deutsche Musik glaubte beherrschen und repräsentieren, sowie seine eigenen Interessen durchsetzen zu können.

Dieses Buch soll aber nicht von Richard Strauss, Paul Hindemith oder Wilhelm Furtwängler, über die bereits viel geschrieben wurde, handeln, sondern bislang wenig beachtete, aber zentrale Aspekte des Musiklebens vor und nach 1933 darstellen (und, soweit möglich, erklären). Ziel der Aufsätze dieses Bandes ist es, allgemein verbreitete Denkfiguren, Argumentationsmuster, Mechanismen und ideologische Implikationen des Musiklebens von 1919 bis 1945 zu verdeutlichen. Das gilt auch dort, wo einzelne Werke behandelt werden, denn der konkrete Zusammenhang beugt in der Beschreibung des Typischen einer rein strukturgeschichtlichen Abstraktion vor.

Wenn im folgenden jeweils von 'Rechten' oder 'Linken' die Rede ist, so ist dies keine Verlegenheitslösung, sondern beruht darauf, daß gerade im Musikleben sich bestimmten Personen politische Richtungen nur vage und ungefähr zuweisen lassen (selbst wenn man weiß, wer im Besitz welchen Parteibuchs war). Die Situation unmittelbar nach dem ersten Weltkrieg macht deutlich, wie problematisch solche Zuweisungen sind, und daB in vieler Hinsicht eher der Konsens im Grundsätzlichen zu betonen ist, als die Differenz im Detail, denn nichtzuletzt auf solche grundsätzlichen, nicht unbedingt parteiabhängigen Argumentationsstrukturen in der Zeit der Republik griffen die Nationalsozialisten nach 1933 zurück. 